\title{
Receiver operating characteristic analysis of prediction for gastric cancer development using serum pepsinogen and Helicobacter pylori antibody tests
}

\author{
Chisato Hamashima ${ }^{1 *}$ D, Shizuka Sasazuki ${ }^{2}$, Manami Inoue ${ }^{2,3}$, Shoichiro Tsugane ${ }^{2}$ and for the JPHC Study Group
}

\begin{abstract}
Background: Chronic Helicobacter pylori infection plays a central role in the development of gastric cancer as shown by biological and epidemiological studies. The H. pylori antibody and serum pepsinogen (PG) tests have been anticipated to predict gastric cancer development.

Methods: We determined the predictive sensitivity and specificity of gastric cancer development using these tests. Receiver operating characteristic analysis was performed, and areas under the curve were estimated. The predictive sensitivity and specificity of gastric cancer development were compared among single tests and combined methods using serum pepsinogen and H. pylori antibody tests.

Results: From a large-scale population-based cohort of over 100,000 subjects followed between 1990 and 2004, 497 gastric cancer subjects and 497 matched healthy controls were chosen. The predictive sensitivity and specificity were low in all single tests and combination methods. The highest predictive sensitivity and specificity were obtained for the serum PG I/II ratio. The optimal PG I/II cut-off values were 2.5 and 3.0. At a PG I/II cut-off value of 3.0, the sensitivity was $86.9 \%$ and the specificity was $39.8 \%$. Even if three biomarkers were combined, the sensitivity was $97.2 \%$ and the specificity was $21.1 \%$ when the cut-off values were 3.0 for PG I/II, $70 \mathrm{ng} / \mathrm{mL}$ for PG I, and $10.0 \mathrm{U} / \mathrm{mL}$ for H. pylori antibody.
\end{abstract}

Conclusions: The predictive accuracy of gastric cancer development was low with the serum pepsinogen and H. pylori antibody tests even if these tests were combined. To adopt these biomarkers for gastric cancer screening, a high specificity is required. When these tests are adopted for gastric cancer screening, they should be carefully interpreted with a clear understanding of their limitations.

Keywords: Gastric cancer, Helicobacter pylori antibody, Serum pepsinogen, Receiver operating characteristic analysis, Cancer screening

\section{Background}

Although the incidence of gastric cancer has decreased worldwide, it remains the fifth most common malignancy in the world [1]. Gastric cancer remains a heavy burden in Eastern Asia, South America, and a number of European countries. However, prevention and screening programs

\footnotetext{
* Correspondence: chamashi@ncc.go.jp

${ }^{1}$ Division of Cancer Screening Assessment and Management, Center for

Social Public Health Sciences, National Cancer Center, 5-1-1 Tsukiji Chuo-ku,

Tokyo 104-0045, Japan

Full list of author information is available at the end of the article
}

for gastric cancer particularly at the national level have not yet been established in most countries. The exceptions are Korea and Japan where gastric cancer screening programs have already been introduced [2]. Recently, the International Agency for Research on Cancer has suggested the establishment of Helicobacter pylori screening and eradication programs in countries with a high incidence of gastric cancer, taking the local context into consideration [3]. However, the efficacy of the screening methods used has not yet been evaluated, although they have been anticipated to reduce gastric cancer incidence. 
Thus, H. pylori screening has not yet been officially introduced either as a national and regional program.

Chronic $H$. pylori infection plays a central role in the development of gastric cancer as shown by biological and epidemiological studies [4]. In a recent study, $H$. pylori infection was reported to be associated with $90 \%$ of non-cardia gastric cancer [5]. The associations of other factors including Cag A, blood type, and lifestyle with gastric cancer have also been investigated [6-11]. Based on several risk factors related to lifestyle, prediction models for gastric cancer have been developed, and these models have demonstrated the capability of discriminating high-risk individuals [12]. The serum pepsinogen (PG) test can diagnose gastric atrophy, and it has been used for gastric cancer screening and risk stratification for gastric cancer with $H$. pylori antibody [13-17]. Sasazuki et al. reported that the odds ratio for gastric cancer development of $H$. pylori infection with the gastric atrophy was higher to that of $H$. pylori infection and this was lower than that of $H$. pylori infection with positive result of CagA [11]. Charvat et al. developed a prediction model for gastric cancer based on $H$. pylori infection and gastric atrophy with the risk factors related to lifestyle [18]. The strong association between gastric cancer and these risk factors suggested a high possibility of predicting gastric cancer incidence in the high-risk group detected by the serum PG and H. pylori antibody tests. If the future risk for gastric cancer development can be optimally clarified, appropriate preventive measures can be taken according to individual risks. These preventive measures can be made more efficient for gastric cancer screening to accurately target cancer screening subjects and decrease the screening frequency of the low-risk group. However, these results are not directly connected with primary cancer screening. To adopt these biomarkers in gastric cancer screening, both sensitivity and specificity should be assessed considering the balance of benefits and harms.

Receiver operating characteristic (ROC) analysis is a widely accepted method for selecting an optimal cut-off value for tests as well as for comparing the sensitivity and specificity of diagnostic tests [19]. Optimal sensitivity and specificity can maintain the balance of the benefits and harms of a diagnostic test. A high possibility of predicting gastric cancer incidence indicates high sensitivity, but the indication of specificity, which identifies the proportion of subjects without gastric cancer, is still unclear. A low specificity reportedly indicates a high false-positive result and this becomes harm in asymptomatic people [20]. Therefore, a high specificity is also required. However, the predictive sensitivity and specificity of these biomarkers for gastric cancer development remain unclear. In this study, we evaluated the predictive sensitivity and specificity of the $H$. pylori antibody and serum PG tests for predicting gastric cancer development by ROC analysis based on a long follow-up period.

\section{Methods \\ Study population}

The Japan Public Health Center (JPHC)-based prospective study on cancer and cardiovascular disease (JPHC study) was established in 1990. The study population was defined as all inhabitants in 27 municipalities under 9 public health centers. The study population and design of the JPHC study have been described in detail elsewhere [11]. As a whole, a population-based cohort of 61,009 men and 62,567 women was identified and followed from January 1, 1990 to December 31, 2004. Blood sample was provided voluntarily by these subjects during their health check-ups and was collected from 1990 to 1995 . Although a questionnaire survey was performed at their health check-ups, there was no question related to medicines for gastric diseases which they were taking. Newly diagnosed cases of cancer were collected through local major hospitals and local cancer registries.

This study was approved by the Institutional Review Board of the National Cancer Center, Japan (Approval number: 2001-013, 14-038). Written informed consent was obtained from all the participants in the JPHC study.

\section{Laboratory data}

The level of IgG antibodies to $H$. pylori was measured using a direct ELISA kit (E.Plate 'Eiken' H. pylori Antibody, Eiken Kagaku Co., Ltd., Tokyo, Japan). The serum levels of PG I and II were measured by two-step enzyme immunoassay using commercial kits (E.Plate 'Eiken' Pepsinogen I and Pepsinogen II Eiken Kagaku Co., Ltd.). All measurements were performed by a person blinded to the study. The levels of PG I, PG II, PG I/II, and $H$. pylori antibody were used for diagnosing and predicting gastric cancer development. In Japan, a combination of PG I, PG I/II, and H. pylori antibody measurements has been a commonly used method for stratifying the risk of gastric cancer. PG I $\leq 70 \mathrm{ng} / \mathrm{mL}$ and PG I/II $\leq 3.0$ indicate chronic atrophic gastritis. $H$. pylori infection was classified as positive when the $H$. pylori antibody titer was $\geq 10 \mathrm{U} / \mathrm{mL}$.

\section{Statistical analysis}

ROC analysis was performed following the Hanley and McNeil's method. The area under the curve (AUC) indicated diagnostic accuracy and defined the optimal cutoff points of the diagnostic tests. The AUC and 95\% confidence interval $(\mathrm{CI})$ were estimated and compared among the different biomarkers or their combination. When the highest likelihood ratio was obtained, the cutoff value for sensitivity and specificity was defined as 
optimal. Statistical analysis was performed using STATA 13.0 (STATA, College Station, TX, USA). All test statistics were two-tailed, and $p$-values $<0.05$ were considered to indicate a statistically significant difference.

Before the main analysis for the prediction of gastric cancer development, ROC analysis for these biomarkers was performed to investigate their ability to diagnose $H$. pylori infection. H. pylori infection was used for determining outcome using 2 cut-off values $(\geq 10 \mathrm{U} / \mathrm{mL}$ and $\geq$ $5 \mathrm{U} / \mathrm{mL}$ ) and the AUCs among PG I, PG II, and PG I/II were compared. Then, gastric cancer was used for determining predictive outcome and the AUCs among PG I, PG II, PG I/II, and H. pylori antibody titer were compared. The AUCs were also compared among combination methods using PG I, PG II, PG I/II, and $H$. pylori antibody. Finally, the AUCs were estimated and compared between the commonly used definition of the defined value of a combination method using PG I, PG I/ II, and $H$. pylori antibody. The subjects were classified into 4 groups according to the risk for gastric cancer development based on their levels of serum PG and $H$. pylori antibody at enrollment. To discriminate the positive and negative results, the following standard categories were used: PG I/II $=3.0$, PG I $=70.0 \mathrm{ng} / \mathrm{mL}$, and $H$. pylori antibody $=10.0 \mathrm{U} / \mathrm{mL}$. Atrophic gastritis was defined on the basis of the results of a combination of PG I/II and PG I. Based on these categories, the results were divided into 4groups. The first group subjects had a "normal" PG level (negative atrophy) and were negative for $H$. pylori antibody (negative $H$. pylori infection). The second group subjects had a "normal" PG level and were positive for $H$. pylori antibody. The third group subjects had an "atrophic" PG level and were positive for $H$. pylori antibody. The fourth group subjects had an "atrophic" PG level and were negative for $H$. pylori antibody.

\section{Results}

\section{Target population}

We used the dataset from a nested case-control study from a large-scale cohort study conducted in Japan (i.e., JPHC study) [11]. The procedure used for the selection of the target population is shown in Fig. 1. Of 97,644 eligible subjects in the JPHC study cohort, 13,467 men and 23,278 women who donated blood samples at baseline were included. Cases of gastric cancer were extracted from the cancer registry for the JPHC study based on site. Among 1,681 cases with a histologically proven diagnosis made from 1990 to 2004, plasma at baseline had been obtained from 512 cases. The case subjects were selected individuals who were diagnosed as having gastric cancer for the follow-up periods based on information from local major hospitals and local cancer registries. For the case subjects, control subjects were selected and matched for sex, age ( \pm 3 years), blood

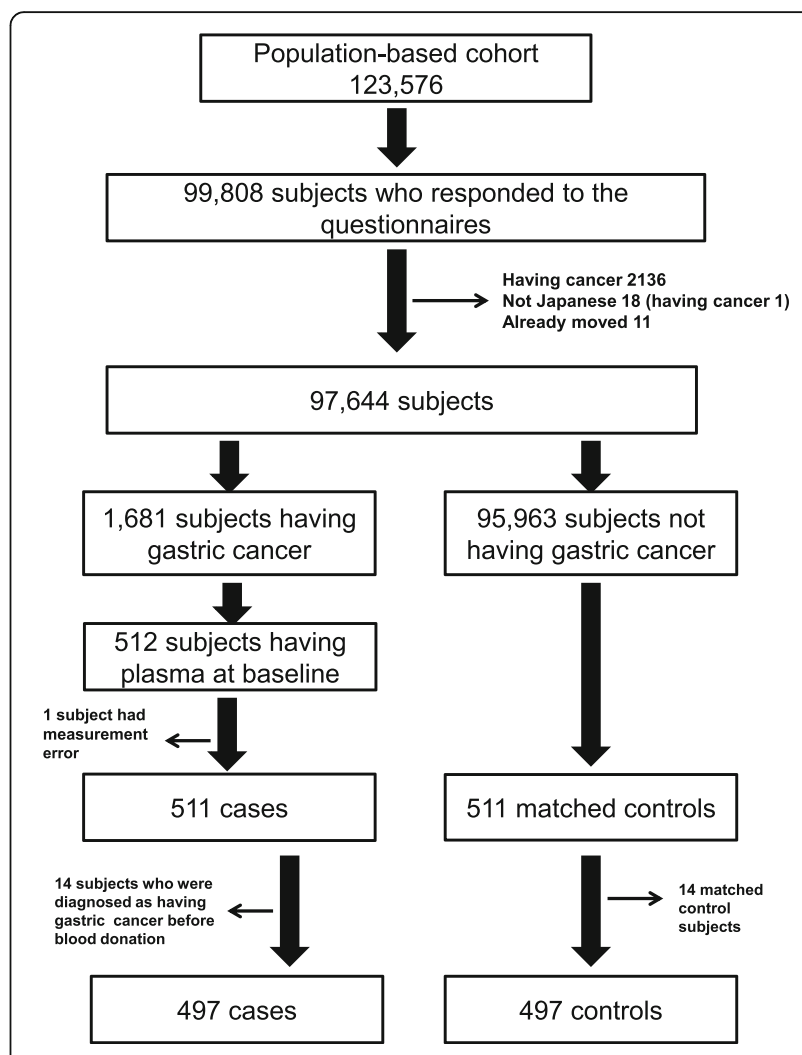

Fig. 1 Flow chart for target group selection. In a large-scale cohort study conducted in Japan (i.e., Japan Public Health Center Study [JPHC study]), a population-based cohort of 61,009 men and 62,567 women was identified and followed from January 1, 1990 to December 31,2004 . Of 97,644 eligible subjects in the JPHC study cohort, 13,467 men and 23,278 women who donated blood samples at baseline were included. Cases of gastric cancer were extracted from the cancer registry for the JPHC study based on site. Among 1,681 cases with a histologically proven diagnosis made from 1990 to 2004, plasma at baseline was obtained from 512 cases. One case with a technical error of $\mathrm{H}$. pylori antibody measurement and the matched control were excluded. The dataset included 511 case subjects and 511 matched control subjects. For this analysis, 14 case subjects who were diagnosed as having gastric cancer before the blood donation date and 14 matched control subjects were excluded. Thus, a total of 994 subjects (497 case subjects having gastric cancer and 497 healthy control subjects) were used for the ROC analysis in this study

donation date ( \pm 2 months), and fasting time at blood donation $( \pm 5 \mathrm{~h})$. One case with a technical error with the H. pylori antibody measurement and the matched control were excluded. The dataset included 511 case subjects and 511 matched control subjects [11]. For this analysis, 14 case subjects who were diagnosed as having gastric cancer before the blood donation date and 14 matched control subjects were excluded. A total of 994 subjects (497 case subjects having gastric cancer and 497 healthy control subjects) were used for the ROC analysis in this study. The proportion of female subjects was $33.6 \%$ and the average age at participation in the cohort 
study was $57.5 \pm 7.2$ years. The average follow-up years from the blood donation date to diagnosis date of gastric cancer for case subjects was $6.1 \pm 3.4$ years.

\section{ROC analysis}

When $H$. pylori infection was classified as a positive case at $\geq 10.0 \mathrm{U} / \mathrm{mL}$, the AUC of PG I/II was $0.820 \pm 0.023$ (95\% CI: 0.774-0.865) which was significantly higher than that of the other biomarkers $(\mathrm{p}<0.001$, Table 1$)$. Even if the cut-off value was changed from $10.0 \mathrm{U} / \mathrm{mL}$ to $5.0 \mathrm{U} / \mathrm{mL}$, a similar result was obtained ( $<<0.001$ ). The optimal values of PG I/II were $95.0 \%$ for sensitivity and $54.8 \%$ for specificity when the $H$. pylori antibody titer was defined as a positive case of $H$. pylori infection at $\geq 10 \mathrm{U} / \mathrm{mL}$.

The AUC of gastric cancer development was higher in PG I/II than in the other biomarkers (Table 1, Fig. 2). The AUC of gastric cancer development was $0.649 \pm$ 0.017 (95\% CI: $0.615-0.683$ ) for PG I/II and $0.574 \pm$ 0.018 (95\% CI: 0.538-0.610) for H. pylori antibody. The optimal cut-off values obtained were 2.5 and 3.0 for PG I/II. When the cut-off value was 2.5 for PG I/II, the optimal values were $71.2 \%$ for sensitivity and $52.5 \%$ for specificity. When the cut-off value was changed to 3.0 for PG I/II, the optimal values were $86.9 \%$ for sensitivity and $39.8 \%$ for specificity.

As PG I/II has the highest AUC among the single tests, the AUCs of PG I/II were compared with those of the other methods (Table 2). The AUC of PG I/II with PG I was always higher than that of PG I/II with PG II or $H$. pylori antibody. When the cut-off value was defined as 3.0 for PG I/II, the optimal cut-off point was $65.0 \mathrm{ng} / \mathrm{mL}$ for PG I and the optimal values were $81.9 \%$ for sensitivity and $42.1 \%$ for specificity. The AUC of the combination of PG I/II and PG I was nearly equal to that of PG I/II $(p=0.2205)$.

The predictive sensitivity and specificity of the combination method using PG I/II, PG I, and H. pylori antibody were estimated with different titers of $H$. pylori antibody from 1.0 to $10.0 \mathrm{U} / \mathrm{mL}$ (Table 3). The cut-off values were defined as 3.0 for PG I/II, $70 \mathrm{ng} / \mathrm{mL}$ for PG I, and $10.0 \mathrm{U} / \mathrm{mL}$ for $H$. pylori antibody. A "normal" PG level (negative atrophy) and negative for $H$. pylori antibody (negative $H$. pylori infection) were defined as negative results. At the standard cut-off value, the sensitivity was $97.2 \%$ and the specificity was $21.1 \%$. When the titer was changed from 10.0 to $3.0 \mathrm{U} / \mathrm{mL}$ for $H$. pylori antibody, the sensitivity increased slightly but the specificity decreased to half. Thus, with a decrease in the titer of $H$. pylori antibody, the AUCs slightly changed. Compared with PG I/II, the AUCs of gastric cancer using the combined method with the standard cut-off values (PG $\mathrm{I} / \mathrm{II}=3.0$, PG I $=70.0 \mathrm{ng} / \mathrm{mL}$, and H. pylori antibody $=$ $10.0 \mathrm{U} / \mathrm{mL}$ ) were nearly equal (Fig. 3). When the PG I/II value was changed from 3.0 to 2.5 and the PG I value was changed from 70.0 to $65.0 \mathrm{ng} / \mathrm{mL}$ in the combination method, the AUCs were similar. The AUCs of gastric cancer development were $0.649 \pm 0.017$ (95\% CI: $0.615-0.683)$ for PG I/II and $0.622 \pm 0.015$ (95\% CI: 0.593-0.651) for the combination method.

\section{Discussion}

The association of various risk factors including Cag A, blood type, and lifestyle with gastric cancer development has been investigated, and several risk factors have been shown to have a strong association [6-11]. Although the predication model has been developed based on these results, methods for risk stratification in connection with gastric cancer screening have not been conclusively identified $[12,18]$. However, the adaptation of the serum PG and H. pylori antibody tests have been anticipated because these methods involve simple blood tests [1317]. A meta-analysis of prospective cohort studies of gastric cancer development using the combination method of $H$. pylori antibody and serum PG tests with gastric cancer screening has shown that it is possible to stratify the background risks of gastric cancer [21]. In this study, we investigated the best available sensitivity and specificity of the serum PG and H. pylori antibody tests for the prediction of gastric cancer development in connection with cancer screening using ROC analysis. However, the AUCs were usually low because the sensitivity was relatively high when the specificity became extremely low. ROC analyses is a graphical technique for

Table 1 Comparison of ROC areas among individual biomarkers

\begin{tabular}{lllll}
\hline $\begin{array}{l}\text { Outcome (Cut-off } \\
\text { value) }\end{array}$ & ROC area $(95 \% \mathrm{Cl})$ & & \\
\cline { 2 - 5 } & $\mathrm{PG} \mathrm{I/II}$ & $\mathrm{PG} \mathrm{I}$ & $0.186 \pm 0.016$ & $\mathrm{HP}$ \\
\hline H. pylori infection & $0.820 \pm 0.023$ & $0.455 \pm 0.022$ & $(0.153-0.217)$ \\
(HP antibody = 10.0) & $(0.774-0.865)$ & $(0.411-0.499)$ & $0.181 \pm 0.018$ & - \\
H. pylori infection & $0.849 \pm 0.024$ & $0.478 \pm 0.025$ & $(0.146-0.216)$ & - \\
(HP antibody = 5.0) & $(0.801-0.896)$ & $(0.429-0.527)$ & $0.434 \pm 0.018$ & $0.574 \pm 0.018$ \\
Gastric cancer & $0.649 \pm 0.017$ & $0.561 \pm 0.011$ & $(0.400-0.469)$ & $(0.538-0.610)$ \\
\hline
\end{tabular}

HP Helicobacter pylori, $P G$ pepsinogen, $R O C$ receiver operating characteristic, $\mathrm{Cl}$ confidence interval 


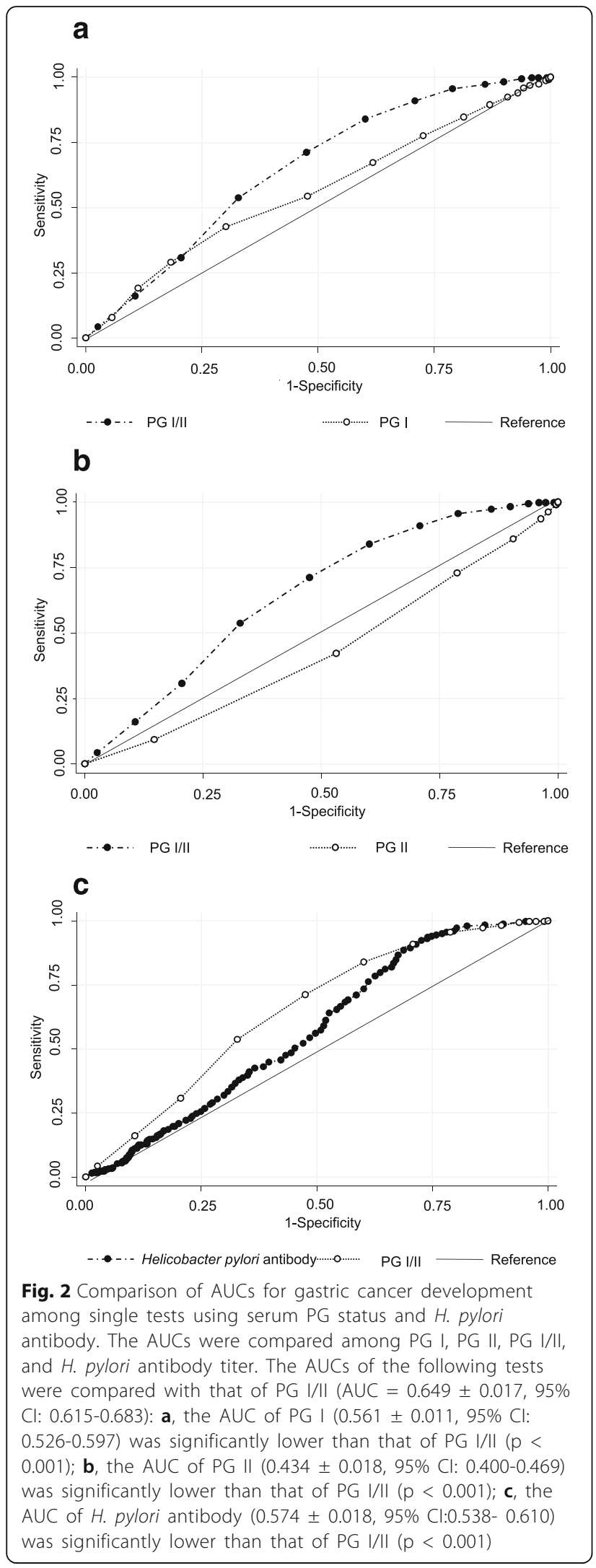

assessing the ability of a test to discriminate between those with disease and those without disease [22]. It allows the determination of the cut-off value at which optimal sensitivity and specificity can be obtained and enables the comparison of 2 or more diagnostic tests. Regarding the interpretation of AUC results, a test with an area $>0.9$ indicates high accuracy, 0.7-0.9 as moderate accuracy, $0.5-0.7$ as low accuracy, and 0.5 as a chance result [22]. In the present $\mathrm{ROC}$ analysis, the AUCs for all the methods were below 0.7 even if the highest AUC was obtained when PG I/II was used as a predictive biomarker for gastric cancer development. Based on these definitions, the predictive sensitivity and specificity of gastric cancer development were found to be low in all single tests and combination methods using serum PG and $H$. pylori antibody. Thus, these biomarkers could not discriminate clearly between individuals with and without gastric cancer development in this study.

When the combination method using serum PG and H. pylori antibody tests was evaluated in this study, a high sensitivity was obtained; however, the specificity was low. In a previous study using the same dataset for a nested case-control study, a strong association between H. pylori infection, gastric atrophy and gastric cancer development was shown. The following odds ratios were obtained when the risk of gastric cancer development was compared with the individuals with both negative $H$. pylori infection and gastric atrophy: 4.2 (95\% CI: 2.2-8.0) for the individuals with positive $H$. pylori infection and negative atrophy; 10.1 (95\% CI: 5.6-18.2) for the individuals with both positive $H$. pylori infection and gastric atrophy; 4.9 (95\% CI: 2.05-12.1) for the individuals with negative $H$. pylori infection and positive gastric atophy [11]. Similar results were obtained from other studies that evaluated the association of $H$. pylori infection, gastric atrophy and gastric cancer development $[11,12,21]$. Although these results confirmed the validity of the strong association for gastric cancer development, and the results supported a high sensitivity for the prediction of gastric cancer development, the possibility of not developing gastric cancer was not assessed and the specificity was ignored. When a prediction model is adopted in clinical practice, it is necessary to provide accurate and discriminating predictions in both situations: with and without gastric cancer development [23]. Therefore, specificity is an important indicator particularity in connection with cancer screening because the target subjects are asymptomatic people. As low specificity translate into an increase in the number of unnecessary examinations, this results in the psychological burden of mislabeling results [20]. When the specificities were calculated on the basis of previous studies which evaluated the association between $H$. pylori infection, gastric 
Table 2 Comparison of ROC areas among the combination of 2 biomarkers

\begin{tabular}{|c|c|c|c|c|}
\hline \multicolumn{2}{|l|}{$P G \mid / / I$} & \multirow{2}{*}{$\frac{P G \mid}{0.627 \pm 0.017}$} & \multirow{2}{*}{$\begin{array}{l}P G \| \\
0.619 \pm 0.017\end{array}$} & \multirow{2}{*}{$\begin{array}{l}\mathrm{HP} \\
0.605 \pm 0.018\end{array}$} \\
\hline 2.5 & ROC area & & & \\
\hline & $(95 \% \mathrm{Cl})$ & $(0.594-0.661)$ & $(0.585-0.652)$ & $(0.571-0.639)$ \\
\hline & Optimal cut-off point & $65 / 60$ & 50 & 5 \\
\hline & Optimal sensitivity & $70.2 / 69.8$ & 71.2 & 70.8 \\
\hline & Optimal specificity & $53.7 / 54.1$ & 52.5 & 54.3 \\
\hline \multirow[t]{5}{*}{3.0} & ROC area & $0.638 \pm 0.017$ & $0.618 \pm 0.017$ & $0.594 \pm 0.018$ \\
\hline & $(95 \% \mathrm{Cl})$ & $(0.604-0.672)$ & $(0.604-0.672)$ & $(0.558-0.629)$ \\
\hline & Optimal cut-off point & 65 & 50 & $5.0 / 6.0$ \\
\hline & Optimal sensitivity & 81.9 & 83.9 & $82.9 / 82.7$ \\
\hline & Optimal specificity & 42.1 & 39.8 & $42.5 / 42.7$ \\
\hline \multirow[t]{5}{*}{3.5} & $\mathrm{ROC}$ area & $0.631 \pm 0.018$ & $0.618 \pm 0.017$ & $0.584 \pm 0.082$ \\
\hline & $(95 \% \mathrm{Cl})$ & $(0.597-0.665)$ & $(0.604-0.672)$ & $(0.548-0.620)$ \\
\hline & Optimal cut-off point & 65 & 50 & $5.0 / 6.0$ \\
\hline & Optimal sensitivity & 88.9 & 91.0 & $89.9 / 89.5$ \\
\hline & Optimal specificity & 31.6 & 29.2 & $33.8 / 34.2$ \\
\hline
\end{tabular}

HP Helicobacter pylori, $P G$ pepsinogen, $R O C$ receiver operating characteristic, $\mathrm{Cl}$ confidence interval

Table 3 Comparison of ROC areas among the combination of 3 biomarkers

\begin{tabular}{|c|c|c|c|c|c|}
\hline \multirow{2}{*}{$\begin{array}{l}\text { HP antibody } \\
\text { values }\end{array}$} & & \multicolumn{2}{|l|}{$P G|/| \mid=3.0$} & \multicolumn{2}{|l|}{$P G I / \|=2.5$} \\
\hline & & $P G I=70$ & PGI = 65 & $P G I=70$ & $P G I=65$ \\
\hline \multirow[t]{4}{*}{1} & $\mathrm{ROC}$ area & $0.619 \pm 0.014$ & $0.623 \pm 0.014$ & $0.623 \pm 0.015$ & $0.625 \pm 0.015$ \\
\hline & $(95 \% \mathrm{Cl})$ & $(0.591-0.646)$ & $(0.595-0.650)$ & $(0.594-0.653)$ & $(0.596-0.655)$ \\
\hline & Sensitivity & 99.4 & 100 & 99.8 & 99.8 \\
\hline & Specificity & 8.7 & 4.4 & 4.6 & 4.6 \\
\hline \multirow[t]{4}{*}{3} & ROC area & $0.620 \pm 0.014$ & $0.624 \pm 0.014$ & $0.630 \pm 0.015$ & $0.632 \pm 0.015$ \\
\hline & $(95 \% \mathrm{Cl})$ & $(0.591-0.648)$ & $(0.596-0.652)$ & $(0.600-0.660)$ & $(0.602-0.662)$ \\
\hline & Sensitivity & 99.0 & 99.2 & 98.6 & 98.6 \\
\hline & Specificity & 11.9 & 11.9 & 12.7 & 12.7 \\
\hline \multirow[t]{4}{*}{5} & ROC area & $0.619 \pm 0.015$ & $0.624 \pm 0.014$ & $0.633 \pm 0.016$ & $0.635 \pm 0.016$ \\
\hline & $(95 \% \mathrm{Cl})$ & $(0.591-0.648)$ & $(0.595-0.652)$ & $(0.603-0.665)$ & $(0.605-0.666)$ \\
\hline & Sensitivity & 98.4 & 98.4 & 97.8 & 97.8 \\
\hline & Specificity & 16.9 & 16.9 & 17.9 & 17.7 \\
\hline \multirow[t]{4}{*}{7} & ROC area & $0.621 \pm 0.015$ & $0.625 \pm 0.015$ & $0.634 \pm 0.016$ & $0.637 \pm 0.016$ \\
\hline & $(95 \% \mathrm{Cl})$ & $(0.593-0.650)$ & $(0.597-0.654)$ & $(0.604-0.664)$ & $(0.606-0.667)$ \\
\hline & Sensitivity & 97.4 & 97.4 & 96.6 & 96.6 \\
\hline & Specificity & 19.1 & 19.1 & 20.1 & 20.1 \\
\hline \multirow[t]{4}{*}{10} & ROC area & $0.622 \pm 0.015$ & $0.626 \pm 0.015$ & $0.636 \pm 0.016$ & $0.638 \pm 0.016$ \\
\hline & $(95 \% \mathrm{Cl})$ & $(0.593-0.651)$ & $(0.597-0.655)$ & $(0.605-0.667)$ & $(0.607-0.669)$ \\
\hline & Sensitivity & 97.2 & 97.2 & 96.4 & 96.4 \\
\hline & Specificity & 21.1 & 21.1 & 22.1 & 22.1 \\
\hline
\end{tabular}




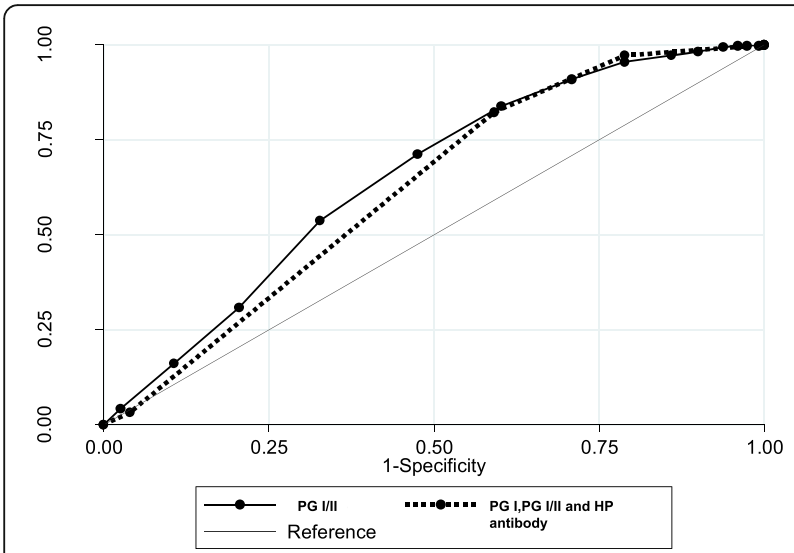

Fig. 3 Comparison of AUCs for gastric cancer development among PG I/II, and combined tests using serum PG status and H. pylori antibody. Compared with PG I/II, the AUCs of gastric cancer using the combined method with the standard cut-off values $(\mathrm{PG} \mathrm{I} / \mathrm{II}=3.0, \mathrm{PG} \mathrm{I}=70.0 \mathrm{ng} / \mathrm{mL}$, and $\mathrm{H}$. pylori antibody $=$ $10.0 \mathrm{U} / \mathrm{mL}$ ) were nearly equal

atrophy, and gastric cancer development, similar results related to sensitivity and specificity were obtained. Based on previous studies related to gastric cancer screening [13-15], the predicative sensitivity and specificity of the combination method using PG I/II, PG I, and H. pylori antibody with a standard cut-off value were $94.0 \%$ and $34.3 \%$, respectively. Even if other risk factors of gastric cancer were included in the model using PG I/II, PG I, and $H$. pylori antibody, the sensitivity and specificity of gastric cancer development were $96.5 \%$ and $28.8 \%$, respectively [18]. Although the basic condition and followup times were different in these studies, the predicative accuracy of gastric cancer development was consistently low using the serum PG and $H$. pylori antibody tests. These results have not been given attention because of the lack of a wide perspective in evaluating the balance of benefits and harms in connection with gastric cancer screening. Thus, only sensitivity was similarly evaluated in these studies.

Prognosis was estimated from the risk of future outcomes in individuals based on their clinical and nonclinical characteristics. Prediction performance could be targeted to a high-risk group for cancer screenings and the use of promotion to encourage participation in the screenings. In the case of low-dose CT screening for lung cancer, risk prediction models have been developed based on different variables including smoking and other risk factors [24-27]. The AUCs of these models were 0.67 to 0.88 and these models discriminated the risk of lung cancer adequately. Although $H$. pylori infection is a primary cause of gastric cancer development, the serum PG and H. pylori antibody tests are insufficient in predicting whether or not an individual has gastric cancer. The aim of an etiological study is to identify particular risk factors attributed to the outcomes. On the other hand, a prediction study provides possible outcomes based on multiple variables associated with the outcome regardless of the cause [28]. In the prediction model, every causal factor is a predictor, but not every predictor is a necessary cause. Because of the possible confusion between an etiological study and a prediction model, biomarkers have been expected to be adopted as cancer screening methods [29, 30]. An accurate prognostic model does not provide any benefits and change the behaviors of the target population of cancer screening if it is not generalizable even though it is verified [31]. In addition, inappropriate use of these biomarkers can lead to a misunderstanding and mismatched labeling of individual risks of cancer. In this study, the highest AUC was obtained in PG I/II, which was also correlated with H. pylori infection. Although the ability of PG I/II to discriminate gastric cancer development is limited, there is another possibility of assessing the appropriate screening interval. In HPV screening for cervical cancer, the screening interval can be expanded after a negative result of HPV testing [32, 33]. The diagnosis of atrophy has improved by conventional endoscopy, thus it has been adopted in clinical practice and endoscopic screening. Nomura et al. have reported that endoscopic findings correlated well with PG I/II based on a multicenter prospective study [34]. Hence, endoscopic diagnosis should also be investigated for the prediction of gastric cancer development in connection with gastric cancer screening. Despite the limitation of PG I/II for predicting gastric cancer development, further study on how to effectively utilize it for gastric cancer screening is advantageous.

This study has several limitations. Firstly, the background of this study has changed compared with that of other studies in the 1990s. The participants then were recruited in the early 1990s for a large-scale cohort study in Japan. Over the last 2 decades, the incidence of gastric cancer and the infection rate of $H$. pylori have decreased, particularly in younger age groups $[35,36]$. Therefore, the present results might not be completely applicable to the current situation. Secondly, the study subjects might not be a representative sample of the whole Japanese population. Our study subjects were taken from the dataset of a previous nested case-control study. The subjects were chosen from 97,644 eligible subjects who participated in the survey and blood donation. In the previous study, the participants in the health check-up survey had different socioeconomic statuses and favorable lifestyle profiles, such as smoking less, participating in more physical exercises, and eating more green vegetables and fruits [37]. Third, we used a casecontrol dataset for this analysis. Diagnostic accuracy can be overestimated if the test is evaluated in a group of 
patients already known to have the disease and in normal patients $[38,39]$. The results might also be overestimated. Fourth, there was no detailed information regarding the medicine the subjects took for gastric disease. As the health insurance did not cover H. pylori eradication during the study period, asymptomatic people had few opportunities to avail of the program. Moreover, a proton pump inhibitor might be also affected by misclassification. Finally, we could not completely exclude individuals with gastric cancer at the baseline because the baseline survey included general health check-up, but not endoscopic examination. Therefore, the predictive sensitivity of gastric cancer development might be overestimated.

\section{Conclusions}

In conclusion, the predictive accuracy of gastric cancer development was low with the serum PG and H. pylori antibody tests even if these tests were combined. To effectively adopt these biomarkers for gastric cancer screening, high specificity and sensitivity are required. When these tests are included for gastric cancer screening, they should be carefully interpreted in terms of their limitations. Further study is needed on how to adopt risk assessment when using these biomarkers and endoscopic diagnosis in connection with gastric cancer screening.

\section{Abbreviations}

AUC: Area under the curve; H.pylori: Helicobacter pylori; JPHC study: Japan Public Health Center (JPHC)-based prospective study; PG: Serum pepsinogen; ROC analyses: Receiver operating characteristic analysis

\section{Acknowledgements}

We are indebted to Dr. Edward F. Barroga (http://orcid.org/0000-0002-89202607) for reviewing and editing the manuscript.

We also thank Ms. Kanoko Matsushima and Ms. Ikuko Tominaga for research assistance.

\section{Funding}

This study was supported by a Grant-in-Aid for H26-Research for Promotion of Cancer Control (Designated study-002) from the Ministry of Health, Labour and Welfare of Japan and the National Cancer Center Research and Development Fund (23-A-31[toku] and 26-A-2; since 2011), by a Grant-in-Aid for Cancer Research from the Ministry of Health, Labour and Welfare of Japan (from 1989 to 2010).

\section{Availability of data and material}

We obtained the data from the Japan Public Health Center (JPHC)-based prospective study on cancer and cardiovascular diseases (JPHC study. The data analyzed for this study are housed at the Epidemiology and Prevention Group, Center for Public Health Science, National Cancer Center, 5-1-1 Tsukiji Chuo-ku, Tokyo 104-0045, Japan. Please contact Dr. Shizuka Sasazuki (ssasuzuki@ncc.go.jp) for any specifics regarding the data used for this study.

\section{Authors' contributions}

$\mathrm{CH}$ and SS designed the study. SS, MI, and ST performed the follow-up survey and collected the data. $\mathrm{CH}$ conducted the statistical analysis of the data. $\mathrm{CH}, \mathrm{SS}, \mathrm{Ml}$, and ST wrote and made critical revisions to the article. All authors read and approved the final manuscript.

\section{Competing interests}

Chisato Hamashima, Shizuka Sasazuki, and Shoichiro Tsugane have nothing to disclose.
Manami Inoue is the beneficiary of a financial contribution from the AXA Research Fund as a chair holder on the AXA Department of Health and Human Security, Graduate School of Medicine, The University of Tokyo. The AXA Research Fund had no role in the design, data collection, analysis, interpretation or manuscript drafting of the article, or in the decision to submit the article for publication.

$\mathrm{Ml}$ is Section Editor and $\mathrm{CH}$ is Associate Editor of BMC Cancer.

Consent for publication

Not applicable.

\section{Ethics approval and consent to participate}

This study was approved by the Institutional Review Board of the National Cancer Center, Japan (Approval number: 2001-013, 14-038). Written informed consent was obtained from all the participants in the JPHC study.

\section{Publisher's Note}

Springer Nature remains neutral with regard to jurisdictional claims in published maps and institutional affiliations.

\section{Author details}

${ }^{1}$ Division of Cancer Screening Assessment and Management, Center for Social Public Health Sciences, National Cancer Center, 5-1-1 Tsukiji Chuo-ku, Tokyo 104-0045, Japan. Epidemiology and Prevention Group, Center for Public Health Sciences, National Cancer Center, 5-1-1 Tsukiji Chuo-ku, Tokyo 104-0045, Japan. ${ }^{3}$ Department of Global Health Policy, Graduate School of Medicine, The University of Tokyo, 7-3-1 Hongo, Bunkyo-ku, Tokyo 113-0033, Japan.

Received: 13 August 2016 Accepted: 4 March 2017

Published online: 09 March 2017

\section{References}

1. International Agency for Research on Cancer: GLOBOCAN. 2012. http://globocan.iarc.fr/. Accessed 10 Aug 2016.

2. Hamashima C. Current issues and future perspectives of gastric cancer screening. World J Gastroenterol. 2014;20:13767-74.

3. IARC Working Group on the Evaluation of Carcinogenic Risks to Humans. HELICOBACTER PYLORI. In: IARC Working Group Reports Volume 8. Helicobacter pylori eradication as a strategy for preventing gastric cancer. Lyon: IARC; 2014. p. 1-4

4. IARC Working Group on the Evaluation of Carcinogenic Risks to Humans. HELICOBACTER PYLORI. In: International Agency of Research on Cancer. IARC Monographs on the evaluation of carcinogenic risks to human: Biological agents. Volume 100B. Lyon: IARC; 2012. p. 385-435.

5. Plummer M, Franceschi S, Vignat J, et al. Global burden of gastric cancer attributable to Helicobacter pylori. Int J Cancer. 2015;136:487-90.

6. Crabtree JE, Covacci A, Farmery SM, et al. Helicobacter pylori-induced interleukin-8 expression in gastric epithelial cells is associated with CagA positive phenotype. J Clin Pathol. 1995:48:41-5.

7. Cover TL, Glupczynski Y, Lage AP, et al. Serologic detection of infection with CagA $^{+}$Helicobacter pyloristrains. J Clin Microbiol. 1995;33:1496-500.

8. Blaser MJ. Intrastrain differences in Helicobacter pylori: a key question in mucosal damage? Ann Med. 1995;27:559-63.

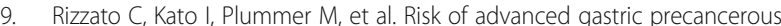
lesions in Helicobacter pylori infected subjects is influenced by ABO blood group and cagA status. Int J Cancer. 2013;133:315-22.

10. Loh JT, Shaffer CL, Piazuelo MB, et al. Analysis of cagA in Helicobacter pylori strains from Colombian populations with contrasting gastric cancer risk reveals a biomarker for disease severity. Cancer Epidemiol Biomarkers Prev. 2011;20:2237-49.

11. Sasazuki S, Inoue M, Iwasaki M, et al. Effect of Helicobacter pylori infection combined with CagA and pepsinogen status on gastric cancer development among Japanese men and women: a nested case-control study. Cancer Epidemiol Biomarkers Prev. 2006;15:1341-7.

12. Buckland G, Travier N, Huerta JM, et al. Healthy lifestyle index and risk of gastric adenocarcinoma in the EPIC cohort study. Int J Cancer. 2015:137:598-606

13. Ohata H, Kitauchi S, Yoshimura N, et al. Progression of chronic atrophic gastritis associated with Helicobacter pylori infection increases risk of gastric cancer. Int J Cancer. 2004;109:138-43. 
14. Watabe $H$, Mitsushima T, Yamaji $Y$, et al. Predicting the development of gastric cancer from combining Helicobacter pylori antibodies and serum pepsinogen status: a prospective endoscopic cohort study. Gut. 2005:54:764-8.

15. Mizuno S, Miki I, Ishida T, et al. Prescreening of a high-risk group for gastric cancer by serologically determined Helicobacter pylori infection and atrophic gastritis. Dig Dis Sci. 2010;55:3132-7.

16. Zhang $X$, Xue $L$, Xing $L$, et al. Low serum pepsinogen I and pepsinogen $|/| \mid$ ratio and Helicobacter pylori infection are associated with increased risk of gastric cancer: 14-year follow up result in a rural Chinese community. Int J Cancer. 2012;130:1614-9.

17. Yoshida T, Kato J, Inoue I, et al. Cancer development based on chronic active gastritis and resulting gastric atrophy as assessed by serum levels of pepsinogen and Helicobacter pylori antibody titer. Int J Cancer. 2014; 134:1445-57.

18. Charvat H, Sasazuki S, Inoue M, JPHC Study Group, et al. Prediction of the 10-year probability of gastric cancer occurrence in the Japanese population: the JPHC study cohort II. Int J Cancer. 2016;138:320-31.

19. Akobeng AK. Understanding diagnostic tests 3: Receiver operating characteristic curves. Acta Paediatr. 2007;96:644-7.

20. Wilt TJ, Harris RP, Qaseem A Screening for cancer: advice for highvalue care from the American College of Physicians. Ann Intern Med. 2015;162:718-25.

21. Terasawa T, Nishida H, Kato K, et al. Prediction of gastric cancer development by serum pepsinogen test and Helicobacter pylori seropositivity in Eastern Asians: A systematic review and meta-analysis. PLoS One. 2014;9, e109783.

22. Fischer JE, Bachmann LM, Jaeschke R. A readers' guide to the interpretation of diagnostic test properties: clinical example of sepsis. Intensive Care Med. 2003:29:1043-51.

23. Royston P, Moons KG, Altman DG, et al. Prognosis and prognostic research: Developing a prognostic model. BMJ. 2009:338:1373-7.

24. Winkler Wille MM, van Riel SJ, Saghir Z, et al. Predictive accuracy of the PanCan lung cancer risk prediction model: External validation based on CT from the Danish Lung Cancer Screening Trial. Eur Radiol. 2015:25:3093-9.

25. Tammemagi CM, Pinsky PF, Caporaso NE, et al. Lung cancer risk prediction: Prostate, Lung, Colorectal and Ovarian Cancer Screening Trial models and validation. J Natl Cancer Inst. 2011;103:1058-68.

26. Kovalchik SA, Tammemagi M, Berg CD, et al. Targeting of low-dose CT screening according to the risk of lung-cancer death. N Engl J Med. 2013;369:245-54.

27. Raji OY, Duffy SW, Agbaje OF, et al. Predictive accuracy of the Liverpool Lung Project risk model for stratifying patients for computed tomography screening for lung cancer: a case-control and cohort validation study. Ann Intern Med. 2012;157:242-50.

28. Moons KG, Royston $P$, Vergouwe $Y$, et al. Prognosis and prognostic research: what, why, and how? BMJ. 2009;338:1317-20.

29. Kondo $\mathrm{H}$, Yoneda M, Inoue $\mathrm{K}$. Application of risk evaluation for gastric cancer ( $A B C$ classification) to population-based gastric cancer screening: Current status and issues in Maniwa City, Okayama Prefecture. J Gastrointestinal Cancer Screen. 2015;53:589-99 (in Japanese).

30. Suzuki H, Saito Y. Current situation of gastric cancer risk evaluation system "ABC classification" in Japanese local governments. J Gastrointestinal Cancer Screen. 2015:53:463-70 (in Japanese).

31. Moons KG, Altman DG, Vergouwe $Y$, et al. Prognosis and prognostic research: application and impact of prognostic models in clinical practice. BMJ. 2009;338:1487-90

32. Dijkstra MG, van Zummeren $M$, Rozendaal $L$, et al. Safety of extending screening intervals beyond five years in cervical screening programmes with testing for high risk human papillomavirus: 14 year follow-up of population based randomised cohort in the Netherlands. BMJ. 2016;355:i4924

33. Elfström KM, Smelov $V$, Johansson AL, et al. Long term duration of protective effect for HPV negative women: follow-up of primary HPV screening randomised controlled trial. BMJ. 2014;348:g130.

34. Nomura S, Ida K, Terao S, et al. Endoscopic diagnosis of gastric mucosal atrophy: multicenter prospective study. Dig Endosc. 2014;26:709-19.

35. Hirayama Y, Kawai T, Otaki J, et al. Prevalence of Helicobacter pylori infection with healthy subjects in Japan. J Gastroenterol Hepatol. 2014;29 Suppl 4:16-9
36. Kamada T, Haruma K, Ito M, et al. Time trends in Helicobacter pylori infection and atrophic gastritis over 40 years in Japan. Helicobacter. 2015;20:192-8.

37. Iwasaki M, Otani T, Yamamoto S, et al. Background characteristics of basic health examination participants: the JPHC Study Baseline Survey. J Epidemiol. 2003;13:216-25.

38. Lijmer JG, Mol BW, Heisterkamp S, et al. Empirical evidence of designrelated bias in studies of diagnostic tests. JAMA. 1999;282:1061-6.

39. Whiting PF, Rutjes AW, Westwood ME, QUADAS-2 Group, et al. QUADAS-2: a revised tool for the quality assessment of diagnostic accuracy studies. Ann Intern Med. 2011;155:529-36.

\section{Submit your next manuscript to BioMed Central and we will help you at every step:}

- We accept pre-submission inquiries

- Our selector tool helps you to find the most relevant journal

- We provide round the clock customer support

- Convenient online submission

- Thorough peer review

- Inclusion in PubMed and all major indexing services

- Maximum visibility for your research

Submit your manuscript at www.biomedcentral.com/submit
Biomed Central 